\title{
Determinants of community-acquired pneumonia in children and young adults in primary care
}

\author{
J. Teepe*, L. Grigoryan\# and T.J.M. Verheij ${ }^{\#}$
}

ABSTRACT: Most studies on determinants of community-acquired pneumonia (CAP) in primary care have focused primarily on the elderly. Using a case-control study in four Dutch healthcare centres, determinants of CAP among children and young adults were identified.

Cases included 156 young adults (aged 16-40 yrs) and 107 children (aged 0-15 yrs) diagnosed with CAP during 1999-2008. For each case, three controls were selected from the same age group. Separate logistic regression analyses were used to identify determinants in young adults and children.

Lower age, asthma and previous upper respiratory tract infections (URTIs) were independently associated with CAP in children. Increasing age, asthma, three or more children at home, current smoking and three or more previous URTIs were independent determinants of CAP in young adults.

The present study has three remarkable findings: 1) increasing age was an independent determinant of CAP in young adults; 2) having young children increased the risk of the development of CAP in young adults; and 3) the number of previous URTIs was independently associated with CAP in both children and young adults, possibly due to higher infection susceptibility. Further studies are required in order to better understand the aetiology of CAP and permit better diagnosis and treatment of this serious condition.

KEYWORDS: Children, community-acquired pneumonia, primary care, risk factors, young adults

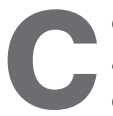
ommunity-acquired pneumonia (CAP) is a common diagnosis and a leading cause of mortality in children and young adults. Its annual incidence is 36 per 1,000 population in children aged $<5$ yrs and $1-8$ per 1,000 population in adults aged 15-44 yrs [1-3]. Pneumonia accounts for approximately a fifth of deaths that occur among children aged $<5$ yrs [4].

Identifying risk factors is important for improving insight into the aetiology of pneumonia and permitting adequate and timely diagnosis. Most risk factors for CAP have been derived from studies that included hospitalised patients, representing only a small proportion of pneumonia cases [5-7]. The few available studies in primary care found increasing age, cigarette smoking, contact with children, chronic bronchitis, asthma, a history of pneumonia and previous respiratory infection to be independent risk factors for CAP $[8,9]$. However, all of these studies focused primarily on elderly patients. In the present study, a restricted number of potentially relevant risk factors for CAP were studied in children and young adults in primary care.

\section{METHODS}

\section{Study population}

A case-control study was conducted in four healthcare centres in a new residential area near the city of Utrecht (the Netherlands). This study was part of the Utrecht Health Project (UHP), which is a health monitoring study in which all new patients that register at one of the four healthcare centres in the area are invited to participate. Participants fill in an individual health profile that includes questionnaires on socioeconomic status, mental health, cardiovascular risk, dietary intake, lifestyle factors (e.g. smoking and alcohol) and housing. The questionnaires are administered by trained research nurses during dedicated interviews. In addition, a standard set of biometric data (including electrocardiogram and lung function) are collected. Disease history and follow-up are registered, using the International Classification of Primary Care (ICPC), in the patient's electronic medical file by general practitioners (GPs). Of the inhabitants in the area, $>60 \%$ are currently participating. The demographic characteristics of the young participants (aged $<40 \mathrm{yrs}$ ) are

\section{AFFILIATIONS}

*University Medical Center Utrecht, and

\# Julius Center for Health Sciences and Primary Care, University Medical Center Utrecht, Utrecht, the Netherlands.

CORRESPONDENCE

L. Grigoryan

Julius Center for Health Sciences and Primary Care

University Medical Center Utrecht Huispost Str 6.131

Postbus 85500

3508 GA Utrecht The Netherlands

E-mail: L.Grigoryan@umcutrecht.nl

Received:

June 292009

Accepted after revision:

Oct 092009 
similar to those of the young Dutch population [10]. All data are gathered continuously in an anonymous central database.

Case ascertainment was based on electronic search. Cases were all adults aged $16-40$ yrs and children aged $<15$ yrs diagnosed with CAP (ICPC code R81) between April 1999 and December 2008. The criteria for the diagnosis of pneumonia (R81) according to ICPHHC-2 were either a confirmation by radiography or the presence of at least three of the following signs / symptoms: 1 ) decreased intensity of breath sounds; 2 ) dullness on chest percussion; 3 ) inspiratory crackles; 4 ) increased vocal resonance; 5) fever ( $\left.\geqslant 38^{\circ} \mathrm{C}\right)$; and 6 ) local chest pain on deep inhalation [11]. All GPs received training in diagnosing pneumonia and ICPC coding.
The controls were adults and children in the same age groups who had not had an episode of CAP during the study period. From the list of patients assigned to the UHP, three controls were randomly selected for each case every year during the study period.

\section{Determinants}

Possible determinants of CAP were selected based on the literature. Data were obtained from the patients' electronic medical files and individual health profiles made by dedicated research nurses during patient registration at the healthcare centres.

Comorbid conditions included asthma (ICPC code R96), upper respiratory tract infections (URTIs; R74), hay fever (R97),

TABLE 1 Analyses of risk factors for community-acquired pneumonia (CAP) in children

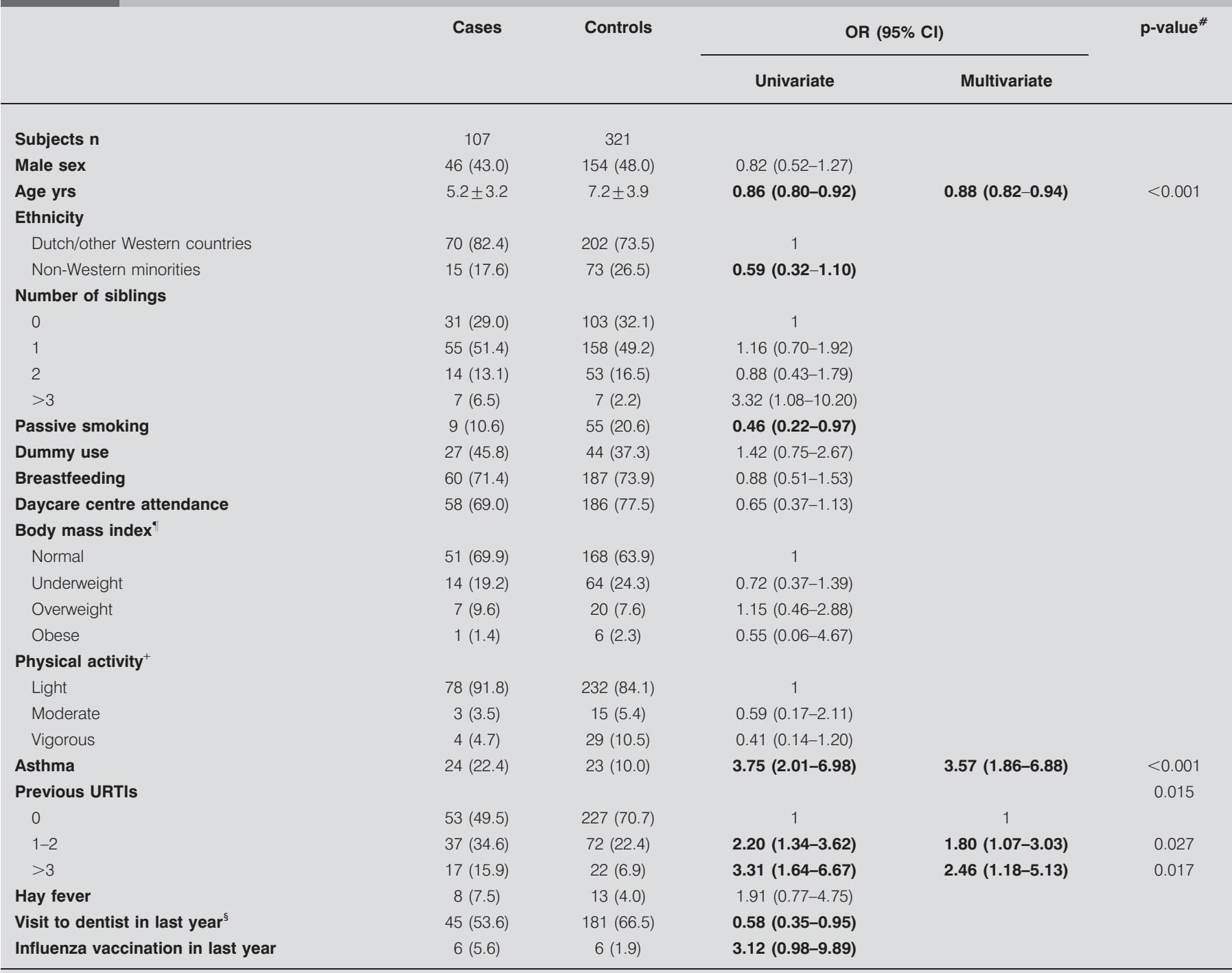

Data are presented as mean \pm SD or $n(\%)$, unless otherwise indicated. The multivariate regression model included all factors with a $p$-value of $<0.10$ in univariate analyses. Results shown in bold type have a p-value of $<0.10$ in univariate analyses and a p-value of $<0.05$ in multivariate analyses; where the univariate data is shown in bold type but there is no corresponding multivariate data, the results were nonsignificant on multivariate analysis. OR: odds ratio; Cl: confidence interval; URTI: upper respiratory tract infection. ${ }^{*}$ : for multivariate analysis; ${ }^{\bullet}$ : in children aged $2-15 \mathrm{yrs}$; ${ }^{+}$: light 0 to $<4.0$ metabolic equivalents of the task (METs), moderate 4.0 to $<6.5$ METs, and vigorous $\geqslant 6.5 \mathrm{METs}$; ${ }^{\text {: }}$ one or more. 
TABLE 2 Analyses of risk factors for community-acquired pneumonia (CAP) in young adults

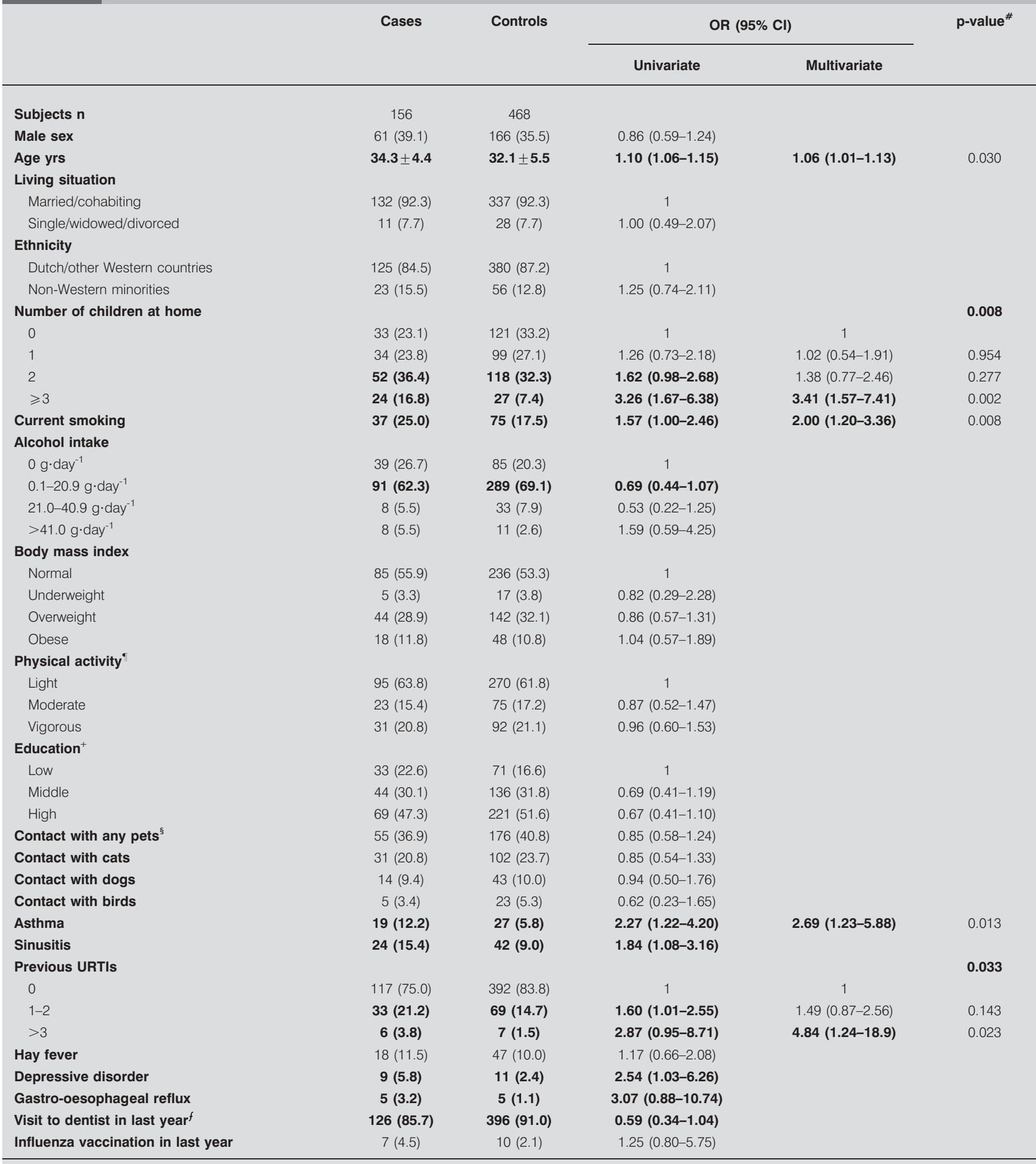

Data are presented as mean \pm SD or $n(\%)$ unless otherwise indicated. The multivariate regression model included all factors with a $\mathrm{p}$-value of $<0.10$ in univariate analyses. Results shown in bold type have a p-value of $<0.10$ in univariate analyses and a p-value of $<0.05$ in multivariate analyses; where the univariate data is shown in bold type but there is no corresponding multivariate data, the results were nonsignificant on multivariate analysis. OR: odds ratio; Cl: confidence interval; URTI: upper respiratory tract infection. " : for multivariate analysis. ": light 0 to $<4.0$ metabolic equivalents of the task (METs), moderate 4.0 to $<6.5 \mathrm{METs}$, and vigorous $\geqslant 6.5 \mathrm{METs}$. ${ }^{+}$low: lower vocational and primary, medium: intermediate vocational and secondary, and high: higher vocational and university. ${ }^{\varsigma}$ : not including fish. ${ }^{f}$ : one or more. 
depressive disorder (P76), gastro-oesophageal reflux (D84/ D90) and sinusitis (R75). The time period during which previous URTIs occurred was 6 yrs for young adults and 7 yrs for children. Information was also extracted on age, sex, current smoking, contact with pets, dental visits during the last year, influenza vaccination during the last year, passive smoking, dummy use and breastfeeding. Passive smoking was defined as daily exposure to tobacco smoke of children at home. Current smoking was defined as smoking at least one cigarette daily. Civil status in young adults was categorised into two groups: married/cohabiting, and single/widowed/ divorced. Ethnicity, according to the birthplace of the patients or their parents, was grouped into Western and non-Western. For children, number of siblings included siblings aged $<10$ yrs. Children at home included children aged $<10$ yrs. Alcohol intake was measured as total daily alcohol consumption in grams. For young adults, body mass index was grouped into four categories: <18.5 (underweight), 18.5-24.9 (normal weight), 25.0-29.9 (overweight), and >30.0 (obese) [12-14]. Physical activity information obtained from the Short QUestionnaire to ASsess Health-enhancing physical activity (SQUASH) was grouped into three categories: light (0 to $<4.0$ metabolic equivalents of the task (METs)), moderate (4.0 to <6.5 METs), and vigorous ( $\geqslant 6.5 \mathrm{METs}$ ) [15]. Education comprised three categories: low (lower vocational and primary), middle (intermediate vocational and secondary), and high (higher vocational and university).

\section{Data analysis}

Since different determinants of CAP were assumed in children and young adults, all analyses were performed separately. The characteristics of cases and controls were compared using univariate logistic regression to calculate odds ratios (ORs) and their $95 \%$ confidence intervals (CIs). Analyses were performed by multivariable logistic regression with CAP as a dependent variable and including factors which had a p-value of $<0.10$ on univariate analysis. Factors were regarded as significant in multivariate analyses when they had a p-value of $<0.05$. Nonsignificant factors from the multivariate analyses were deleted from the model stepwise. Possible interactions between factors found to be significant in the multivariable analyses were tested and the significance set at a p-value of $<0.01$ for interaction terms due to multiple testing. Data were analysed using SPSS (version 15) for Windows (SPSS, Inc., Chicago, IL, USA).

\section{RESULTS}

The present study included 107 CAP cases and 321 controls among children, and 156 cases and 468 controls among young adults. Demographic data and the results of univariate and multivariate analyses among children and young adults are shown in tables 1 and 2, respectively.

Asthma was the strongest independent risk factor for CAP in children (OR 3.57, 95\% CI 1.86-6.88) and young adults (OR $2.69,95 \%$ CI 1.23-5.88). Previous recurrent URTIs were another independent risk factor for CAP in children and young adults. For young adults, increasing age was an independent risk factor for CAP (OR 1.06, 95\% CI 1.01-1.13), whereas, for children, it was a protective factor. Other independent risk factors in young adults were current smoking (OR 2.00, 95\% CI
1.20-3.36) and having three or more children at home (OR 3.41, $95 \%$ CI 1.57-7.41). No statistical interaction was found between the determinants of CAP in the multivariable analyses.

\section{DISCUSSION}

To our knowledge, this is the first study exploring the determinants of CAP in children and young adults in primary care. For children, a history of asthma, previous URTIs and lower age were independent determinants. For young adults, independent determinants were a history of asthma, number of children at home, previous URTIs, current smoking and increasing age.

Other studies including older patients also confirmed age as a risk factor for CAP $[5,9]$. Notably, the present results showed that increasing age is a risk factor even in young adults (aged 16-40 yrs). A possible explanation could be that older adults, i.e. those closer to the age of $40 \mathrm{yrs}$, have children living in the household more frequently and are, therefore, more frequently exposed to infections of their children. Regular contact with young children was an independent risk factor for CAP in two other studies $[8,16]$. In the present study, having three or more children in the household was also significantly associated with CAP in young adults. However, multivariable analysis showed that the relationship found between age and CAP was independent of the number of children in the household. Waning immunity, even in this age group, could be an explanation for this finding. The study also showed a clear relation between the occurrence of CAP and an increasing number of previous URTIs. Higher infection susceptibility in these patients could be a possible mechanism causing predisposition to pneumonia.

Some potential limitations of the present study should be taken into account. As the sample size was limited, those factors that have a weak association with CAP might not have been detected. However, whether such weaker associations are clinically relevant is uncertain. Another potential limitation is that the diagnosis of pneumonia was not confirmed by radiographic findings in all cases. Conversely, this reflects empirical diagnosis in daily practice in primary care and thus enhances the generalisability of the results. Finally, information on previous CAP was not available and so this could not be included as a possible determinant.

In conclusion, apart from confirming well-known risk factors, such as smoking, asthma and contact with young children, the present study showed that, even in young adults, increasing age and number of previous URTIs were related to the occurrence of CAP. Further studies are required in order to better understand the role of host factors in the aetiology of CAP and thus permit better diagnosis and treatment of this important disease in children and young adults.

\section{STATEMENT OF INTEREST}

None declared.

\section{REFERENCES}

1 Foy HM, Cooney MK, Allan I, et al. Rates of pneumonia during influenza epidemics in Seattle, 1964 to 1975. JAMA 1979; 241: 253-258. 
2 Jokinen C, Heiskanen L, Juvonen $\mathrm{H}$, et al. Incidence of communityacquired pneumonia in the population of four municipalities in eastern Finland. Am J Epidemiol 1993; 137: 977-988.

3 Almirall J, Bolibar I, Vidal J, et al. Epidemiology of communityacquired pneumonia in adults: a population-based study. Eur Respir J 2000; 15: 757-763.

4 Bryce J, Boschi-Pinto C, Shibuya K, et al. WHO estimates of the causes of death in children. Lancet 2005; 365: 1147-1152.

5 Farr BM, Bartlett CL, Wadsworth J, et al. Risk factors for community-acquired pneumonia diagnosed upon hospital admission. Respir Med 2000; 94: 954-963.

6 Falguera M, Carratala J, Ruiz-Gonzalez A, et al. Risk factors and outcome of community-acquired pneumonia due to Gramnegative bacilli. Respirology 2009; 14: 105-111.

7 Kang CI, Song JH, Oh WS, et al. Clinical outcomes and risk factors of community-acquired pneumonia caused by Gram-negative bacilli. Eur J Clin Microbiol Infect Dis 2008; 27: 657-661.

8 Almirall J, Bolibar I, Serra-Prat M, et al. New evidence of risk factors for community-acquired pneumonia: a population-based study. Eur Respir J 2008; 31: 1274-1284.

9 Farr BM, Woodhead MA, Macfarlane JT, et al. Risk factors for community-acquired pneumonia diagnosed by general practitioners in the community. Respir Med 2000; 94: 422-427.
10 Grobbee DE, Hoes AW, Verheij TJ, et al. The Utrecht Health Project: optimization of routine healthcare data for research. Eur J Epidemiol 2005; 20: 285-287.

11 World Organization of Family Doctors International Classification Committee, ICPC-2. International Classification of Primary Care. 2nd Edn. Oxford, Oxford University Press, 1998.

12 World Health Organization. Global Database on Body Mass Index. BMI Classification. www.who.int/bmi/index.jsp?introPage $=$ intro_3.html Date last updated: June 7, 2009. Date last accessed: June 7, 2009.

13 Cole TJ, Bellizzi MC, Flegal KM, et al. Establishing a standard definition for child overweight and obesity worldwide: international survey. BMJ 2000; 320: 1240-1243.

14 Cole TJ, Flegal KM, Nicholls D, et al. Body mass index cut offs to define thinness in children and adolescents: international survey. BMJ 2007; 335: 194.

15 Wendel-Vos GC, Schuit AJ, Saris WH, et al. Reproducibility and relative validity of the Short Questionnaire to Assess Healthenhancing physical activity. J Clin Epidemiol 2003; 56: 1163-1169.

16 Schnoor $\mathrm{M}$, Klante $\mathrm{T}$, Beckmann $\mathrm{M}$, et al. Risk factors for community-acquired pneumonia in German adults: the impact of children in the household. Epidemiol Infect 2007; 135: 1389-1397. 